\title{
CONSPECTVS SIGLORVM ET NOTARVM
}

P, $\pi$ cod. Vatican. Gr. 190, s. X

B cod. Bodleian. Dorvillian. 10, 1, s. IX

F, $\varphi$ cod. Florentin. Laurentian. 28, 3, s. X

v cod. Vindobon. Gr. 103, s. XI-XII

b cod. biblioth. comm. Bononiensis 18-19, s. XI

p cod. Parisin. Gr. 2466, s. XII

\begin{tabular}{|c|c|c|}
\hline$\pi \alpha \varrho a ́ \lambda \lambda \eta \eta \lambda \circ \iota v \dot{v} \vartheta \varepsilon i a \iota$ & $\|$ & parallelae rectae \\
\hline 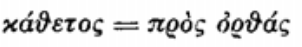 & $\perp$ & perpendicularis \\
\hline$\gamma \omega v i a$ & $\angle$ & angulus \\
\hline$\gamma \omega v i \alpha$ ò $\vartheta \dot{\eta}$ & $L$ & angulus rectus \\
\hline$\tau \varrho i \gamma \omega \nu o v$ & $\Delta$ & triangulum \\
\hline$\tau \varepsilon \tau \varrho a ́ \gamma \omega \nu o v$ & $\square$ & quadratum \\
\hline$\pi \alpha \varrho \alpha \lambda \lambda \eta \lambda \delta ́ \gamma \varrho \alpha \mu \mu о \nu$ & $\square$ & parallelogrammum \\
\hline$\tau \varrho a \pi \varepsilon \zeta \zeta \iota v$ & $\square$ & trapezium \\
\hline ögos & & definitio (= def.) \\
\hline 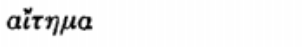 & & postulatum ( $=$ post.) \\
\hline 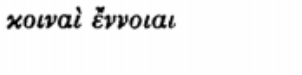 & & $\begin{array}{l}\text { communes animi conceptiones } \\
(=\text { comm. a. conc. })\end{array}$ \\
\hline 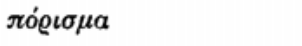 & & coroliarium $(=$ coroll. $)$ \\
\hline$\vartheta \varepsilon \omega ́ \varrho \eta \mu \alpha$ & & propositio (= prop.) \\
\hline
\end{tabular}

[ ] delendum aliquid in textu indicant 